\title{
THE EFFECT OF HEAT AND MICROWAVE TREATMENTS ON ORANGE JUICE QUALITY DURING STORAGE
}

\author{
Thamer A. Khalil
}

Department of Food Science, College of Agriculture and Forestry, Mosul

University, Iraq.

Emil:thamerkhalil@yahoo.com

\begin{abstract}
The effect of heat and microwave (MW) treatments on quality related parameters as ascorbic acid (AA), browning index (BI), reducing sugar (RS), total soluble solid percentage (TSS \%), and $\mathrm{pH}$ of orange juice (OJ) were investigated directly after the treatments and after storage at 4 and $20{ }^{\circ} \mathrm{C}$ for 2 months. Microwave treatment induced little reduction in AA compared to heat treatment. Microwave treatment ensured the quality stability of orange juice stored for 2 months under refrigeration $\left(4{ }^{\circ} \mathrm{C}\right)$ but inferior orange juice quality was detected after 2 month storage at $20{ }^{\circ} \mathrm{C}$. Using MW exposure above $60 \mathrm{sec}$ gave better studied parameters than heat treatments. However, microwave exposure for more than $60 \mathrm{sec}$. was sufficient to preserve the OJ quality. Accordingly, the use of microwave energy may be proposed as an alternative to traditional heat treatment in order to preserve the OJ quality.
\end{abstract}

Keywords: microwave, heat treatment, orange juice, ascorbic acid, browning index.

Received: 28/5/2012, Accepted: 6/5/2013

\section{INTRODUCTION}

Orange juice is one of the most popular beverages in Iraq as well as in the worldwide. When OJ is stored, it gradually turns brown. It is well known that OJ is rich in AA. The nutritional value of OJ is related primarily to the content of AA. Two of the major changes during storage of OJ are development of off-flavour and wning (Handwerk and Coleman, 1988). However, AA is also known to contribute to browning of some foods because it is easily oxidized and decomposed. The decomposition of AA which leads to nonenzymatic browning, is the main deteriorative phenomena that occurs during storage of OJ. Tatum et al. $(1969,1975)$ showed several degradation products of AA during storage of OJ. On the other hand, it was reported that AA most contributes to browning within two weeks of storage (Shinoda et al., 2004). However, it has been shown that the browning occurred in acidic fruit juices during storage was attributed mainly to nonenzymatic reactions (Burdurlu and Karadeniz, 2003, Khalil and Al-Zubaidy, 2010).

Commercial OJ has been traditionally heat-processed to destroy spoiling microorganisms and inactivate enzymes that curb the product quality during storage (Braddock, 1999). Heat treatment often induces undesirable changes in the colour, flavour and nutritional value of OJ (Giner et al., 2003). Negative effects of thermal treatment include nonenzymatic browning, loss of nutrients and formation of undesirable products such as 5-hydroxymethylfurfural (5-HMF). Browning due to the thermal treatment is the result of several reactions known as Maillard reactions, which include condensation between RS and amino acids, caramellization, AA 
browning and pigment destruction (Beveridge et al., 1986; Cornwell and Wrolstad 1981). However, such processing generally involves heat treatment that can reduce organoleptic quality (Damasceno et al. 2008). Accordingly, researchers have emphasized to optimize the time/temperature profiles to minimize the exposure of food to heat. In order to reduce the negative effects of heat treatments in foods, alternative technologies capable of reducing the deteriorating effect at temperatures below those used during thermal treatment are being demanded by the food industry (Kozempel et al., 2000). Therefore non-thermal food treatment techniques are receiving considerable attention because of their potential to reduce or even eliminate heat exposure. Orange juice has become a strong candidate for the application of non thermal processes due to degradation of fresh flavour characteristics by the thermal processes currently in use for ready to drink products, such as pasteurization. Electromagnetic heating, on the other hand, has been successfully used for the efficient pasteurization of food products in the recent years. MW heating is a process within a family of electromagnetic techniques such as induction, radio frequency, direct resistance or infra-red heating. Moreover, using MW for industrial treatment are rapid heat transfer, volumetric and selective heating, compactness of equipment, speed of switching on and off and pollutionfree environment as there are no products of combustion (Metaxas, 1996; Metaxas and Meredith, 1993). Several studies have successfully been carried out into the MW pasteurization of fruit juices, as it preserves the natural organoleptic characteristics of the juice and reduces the time of exposure to energy, with the subsequently lower risk of losing essential thermolabile nutrients (Canumir et al., 2002). The objective of the present study is to examine the effect of MW treatment on OJ preservation in comparison with heat treatment.

\section{MATERIALS AND METHODS}

Sampling preparation: the fresh sweet orange (Citrus sinensis, origin Chili) used for this investigation were purchased from the local fruit market. The orange fruits were washed with running water and pressed manually using a plastic bowl juicer. The produced juice was filtered through muslin cloth.

Thermal treatments: the thermal treatment was carried out for the OJ in conical flasks of $500 \mathrm{~mL}$ volume at four different temperatures $70,80,90$ and $95{ }^{\circ} \mathrm{C}$ for different periods $2,4,6,8$, and 10 minutes using a thermostatic water bath. The treated samples were immediately brought to room temperature in an ice-water bath. $20 \mathrm{~mL}$ of each treated juice sample was put in sterilized, tightly screwed, and opaque test tubes $\left(250 \mathrm{~mm}\right.$ length and $15 \mathrm{~mm}$ diameter) and stored at 4 and $20^{\circ} \mathrm{C}$.

Microwave treatment: the MW oven (Model No. NMO-502N, Nikayajapan LTD, Kobe, Japan) at $2450 \mathrm{MHz}$ (based on US Federal Communication Commission) was used for different periods 30, 60, 90, 120, and 150 seconds. A sterilized glass tubes (250 mm length and $15 \mathrm{~mm}$ diameter) were inserted and an automatic program that controls MW incidence was applied. Maximum volume of $20 \mathrm{~mL}$ was used to obtain homogeneity of MW incidence. The treated samples were kept in the above mentioned test tubes as tightly screwed and stored under opaque condition at $4{ }^{\circ} \mathrm{C}$ and $20^{\circ} \mathrm{C}$. The temperature of MW-treated samples was raised as affected by the time of exposure of microwaving as follows: 
Mesopotamia J. of Agric. Vol. (46) No. (3) 2018
ISSN: 2224 - 9796 (Online) ISSN: 1815 - 316 X (Print)

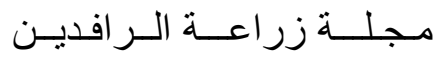
المجلد (46) العدد (3) 2018

$\begin{array}{lrrrrr}\text { Exposure time (sec.): } & 30 & 60 & 90 & 120 & 150 \\ \text { Temperature }\left({ }^{\circ} \mathrm{C}\right): & 37 & 48 & 60 & 85 & 98\end{array}$

Physical and chemical analysis

Ascorbic acid: the quantitative analysis of AA were carried out by using the sensitive spectrometric method adopted by Revanasiddappa and Veena (2008) for control and treated juice samples on day 0 and after 2 months of storage at $4{ }^{\circ} \mathrm{C}$ and $20^{\circ} \mathrm{C}$. The absorbance of the treated and control samples were measured at $550 \mathrm{~nm}$ (using a spectrophotometer (Model 6300, Jenway, U. K,) against distilled water. The blank was prepared similarly by omitting the AA and its absorbance was measured against distilled water. The difference in absorbance values was used for constructing the calibration curve.

Colour assessment: the browning colour of control and treated juice samples was measured as BI according to the method described by Ranganna (1977) using a spectrophotometer (Model 6300, Jenway, U. K,) at $420 \mathrm{~nm}$, on a day 0 and 2 month storage at $4{ }^{\circ} \mathrm{C}$ and $20{ }^{\circ} \mathrm{C}$.

Reducing sugar: total juice RS undertaken as glucose and fructose was measured spectrophotometrically according to Miller (1972) using a spectrophotometer (Model Jenway, U.K.) at $575 \mathrm{~nm}$.

Determination of $\mathbf{p H}$ and total soluble solids content: the $\mathrm{pH}$ was measured by using a portable pH-meter (Model SN 08/4521 Lovibond, SensoDirect, Germany) while TSS \% were determined by using a portable Abbe-refractometer (Abbe Atago, Japan).

Statistical analyses: CRD experimental design with two repetitions was applied. Data were analyzed using the Statistical Analysis System (SAS Institute, Cary, NC, USA) for the analysis of variance (ANOVA).

\section{Heat treatment of orange juice samples}

\section{RESULTS AND DISCUSSION}

Table 1 shows the physicochemical parameters of freshly squeezed OJ. At time zero (T0), different heat treatments showed fluctuation in various parameters studied on the OJ samples (Table 2). In general, there were wide variations in the AA content of the fresh juice $(36 \pm 28.54 \mathrm{mg} / 100 \mathrm{~mL})$. This indicated that the heat treatment method had a definite influence on the retention of AA. In addition, the mean values showed that temperature had a greater influence and the degradation was rapid at higher temperatures. The temperature and time profiles for different heat treatments at T0 affecting AA content was indicated in Fig. 1.

Table (1): Characteristics of the fresh orange juice

\begin{tabular}{|c|c|}
\hline Ascorbic acid $(\mathrm{mg} / 100 \mathrm{~mL})$ & 36 \\
\hline Browning index $($ Abs. at $420 \mathrm{~nm})$ & 0.033 \\
\hline Total soluble solids \% & 10.8 \\
\hline $\mathrm{pH}$ & 2.95 \\
\hline Reducing sugar $(\mathrm{gm} / 100 \mathrm{~mL})$ & 5 \\
\hline
\end{tabular}

The highest AA retention $(20.41 \mathrm{mg} / 100 \mathrm{~mL})$ was observed when heating OJ at $70{ }^{\circ} \mathrm{C}$ for $2 \mathrm{~min}$. On the other hand, the lowest AA obtained $(7.46 \mathrm{mg} / 100 \mathrm{~mL})$ was experienced during the highest heat treatment used $\left(95^{\circ} \mathrm{C} / 10 \mathrm{~min}\right.$.). Several 
studies emphasized the fact that the more sever heat treatment of fruit juices the more degradation of nutrients, and ascorbic acid in particular (Khalil and AlZubaidy, 2010; Vikram et al., 2005; Lima et al., 1999; Dio Alvarado and Viteri, 1991). In accordance with this finding it can be easily noticed that the degradation of AA, as related to heat treatment, was coincided with the increase of nonenzymatic browning in treated juice samples (Table 2). Nonenzymatic browning caused by elevated heat treatment, can induce browning reaction precursors such as Maillard reaction, caramellization, and AA degradation of which in turn they produce the browning pigment products (Shinoda et al. 2004) and they can be expressed by the BI determination. It can be observed that there is an increase of BI immediately after the heat treatments (T0) experienced in this study (Fig. 1b), however, the values of BI were increased with elevating the heat treatment. The mean vales of RS (at the range of 4.74-4.79 gm/100 mL), TSS (at the range of 10.5$10.7 \%$ ), and $\mathrm{pH}$ (at the range of 2.91-2.94) have been less affected after immediate heat treatments (T0) studied (Table 2). Generally, temperature and time effects have had less significant on RS, pH, and TSS \% rather than on AA and BI of treated OJ. Similar results were found by Elez-Martınez et al. (2006), and Kim and Tadini (1999) who showed that temperature and holding time had no effect on $\mathrm{pH}$ and Brix $^{\circ}$ of conventional pasteurized juice. The storage temperature and period of heattreated juice samples had a great influence on the parameters studied (Table 3,4). $\mathrm{AA}$ and RS values among other values were the most affected by the storage conditions. Nevertheless, 2 month storage of heat-treated juice samples at $4{ }^{\circ} \mathrm{C}$ had somehow less effect on OJ quality. It can be elucidated that the nonenzymatic browning reaction was more activated in higher storage temperature. In addition, the increased degradation of AA and less RS content due to increasing storage temperature have been responsible for higher BI. The visual colour as expressed by $\mathrm{BI}$ and the degradation of AA for all heat-treated juice samples stored at $4{ }^{\circ} \mathrm{C}$ and $20{ }^{\circ} \mathrm{C}$ are shown in Fig. 2 and Fig. 3 respectively. Although a significant reduction in the parameters studied $(\mathrm{p}<0.05)$ of heat-treated OJ samples, storing heat-treated juice samples at $4{ }^{\circ} \mathrm{C}$ had a superior quality over those stored at $20{ }^{\circ} \mathrm{C}$. However, storage at $4{ }^{\circ} \mathrm{C}$ of heat-treated samples for either 2 or 4 minutes at $70{ }^{\circ} \mathrm{C}$ showed more stability of juice quality than other experimented heat treatments. Similar approach was found by Snir et al.(1996) and Elez-Martınez et al. (2006) who carried out the heat treatment at $70{ }^{\circ} \mathrm{C}$ and $5 \mathrm{~min}$ on citrus juices. Nevertheless, they are high enough to obtain good quality products with a convenient juice stabilization, which will be kept under refrigeration conditions. Further, Igual et al. (2010) stated that the $\mathrm{Brix}^{\circ}$ and $\mathrm{pH}$ of pasteurized grapefruit juices were never affected when stored under refrigeration condition. On the other hand, it was suggested that the browning colour derived from the degradation of AA is strongly affected by the RS or their decomposition products (Shinoda et al., 2004). Relatively it can be noticed that the increase in BI values as affected by different storage conditions, was well correlated with the higher reduction of both AA and RS contents (Table 3,4 ), and was more pronounced in storage at $20{ }^{\circ} \mathrm{C}$ than $4{ }^{\circ} \mathrm{C}$. 
Mesopotamia J. of Agric.

Vol. (46) No. (3) 2018

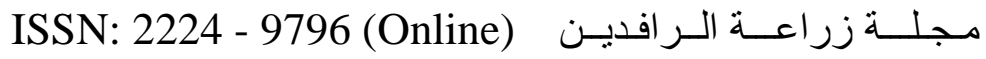
ISSN: 1815 - 316 X (Print) 2018 المجلد (46) العدد (3) اعرد

Table (2): The effect of heat treatment and time on fresh orange juice

\begin{tabular}{|c|c|c|c|c|c|c|}
\hline \multirow[t]{2}{*}{ Determination } & \multirow[t]{2}{*}{ Time (min.) } & \multicolumn{4}{|c|}{ Temperature $\left({ }^{\circ} \mathrm{C}\right)$} & \multirow[t]{2}{*}{ Mean } \\
\hline & & 70 & 80 & 90 & 95 & \\
\hline \multirow{6}{*}{$\begin{array}{l}\text { Ascorbic acid } \\
(\mathrm{mg} / 100 \mathrm{~mL})\end{array}$} & 2 & 20.41 & 16.31 & 14.47 & 13.45 & $16.16^{\mathrm{a}}$ \\
\hline & 4 & 19.09 & 18.53 & 13.85 & 11.33 & $15.70^{\mathrm{a}}$ \\
\hline & 6 & 16.44 & 14.52 & 12.18 & 10.46 & $13.40^{\mathrm{b}}$ \\
\hline & 8 & 14.73 & 13.47 & 11.53 & 8.40 & $12.03^{\mathrm{c}}$ \\
\hline & 10 & 12.61 & 11.34 & 10.86 & 7.46 & $10.57^{\mathrm{d}}$ \\
\hline & Mean & $16.66^{\mathrm{a}}$ & $14.83^{b}$ & $12.58^{\mathrm{c}}$ & $10.22^{\mathrm{d}}$ & \\
\hline \multirow{6}{*}{$\begin{array}{l}\text { Browning index } \\
\text { (Abs. at } 420 \mathrm{~nm} \text { ) }\end{array}$} & 2 & 0.055 & 0.056 & 0.056 & 0.070 & $0.059^{c}$ \\
\hline & 4 & 0.053 & 0.064 & 0.057 & 0.074 & $0.062^{\mathrm{bc}}$ \\
\hline & 6 & 0.052 & 0.066 & 0.060 & 0.077 & $0.064^{\mathrm{ab}}$ \\
\hline & 8 & 0.053 & 0.065 & 0.063 & 0.083 & $0.066^{\mathrm{ab}}$ \\
\hline & 10 & 0.056 & 0.068 & 0.061 & 0.085 & $0.067^{\mathrm{a}}$ \\
\hline & Mean & $0.054^{\mathrm{d}}$ & $0.064^{\mathrm{b}}$ & $0.059^{\mathrm{c}}$ & $0.078^{\mathrm{a}}$ & \\
\hline \multirow{6}{*}{$\begin{array}{l}\text { Reducing sugar } \\
\text { (gm/100 mL) }\end{array}$} & 2 & 4.80 & 4.80 & 4.60 & 4.75 & $4.74^{\mathrm{a}}$ \\
\hline & 4 & 4.80 & 4.75 & 4.80 & 4.80 & $4.79^{\mathrm{a}}$ \\
\hline & 6 & 4.80 & 4.85 & 4.75 & 4.75 & $4.79^{\mathrm{a}}$ \\
\hline & 8 & 4.75 & 4.70 & 4.80 & 4.90 & $4.74^{\mathrm{a}}$ \\
\hline & 10 & 4.75 & 4.85 & 4.75 & 4.65 & $4.75^{\mathrm{a}}$ \\
\hline & Mean & $4.78^{\mathrm{a}}$ & $4.75^{\mathrm{a}}$ & $4.74^{\mathrm{a}}$ & $4.78^{\mathrm{a}}$ & \\
\hline \multirow{6}{*}{$\begin{array}{l}\text { Total soluble } \\
\text { solids (\%) }\end{array}$} & 2 & 10.8 & 10.5 & 10.7 & 10.8 & $10.7^{\mathrm{a}}$ \\
\hline & 4 & 10.7 & 10.4 & 10.4 & 10.9 & $10.6^{\mathrm{ab}}$ \\
\hline & 6 & 10.5 & 10.3 & 10.4 & 10.9 & $10.5^{\mathrm{b}}$ \\
\hline & 8 & 10.5 & 10.3 & 10.8 & 10.9 & $10.6^{\mathrm{ab}}$ \\
\hline & 10 & 10.5 & 10.2 & 10.8 & 11.0 & $10.6^{\mathrm{ab}}$ \\
\hline & Mean & $10.6^{b}$ & $10.3^{\mathrm{c}}$ & $10.6^{\mathrm{b}}$ & $10.9^{\mathrm{a}}$ & \\
\hline \multirow{6}{*}{$\mathrm{pH}$} & 2 & 2.91 & 2.87 & 2.92 & 2.96 & $2.91^{\mathrm{b}}$ \\
\hline & 4 & 2.91 & 2.93 & 2.95 & 2.95 & $2.93^{\mathrm{a}}$ \\
\hline & 6 & 2.92 & 2.93 & 2.95 & 2.94 & $2.93^{\mathrm{a}}$ \\
\hline & 8 & 2.92 & 2.94 & 2.96 & 2.95 & $2.94^{\mathrm{a}}$ \\
\hline & 10 & 2.93 & 2.95 & 2.96 & 2.94 & $2.94^{\mathrm{a}}$ \\
\hline & Mean & $2.92^{b}$ & $2.92^{b}$ & $2.95^{\mathrm{a}}$ & $2.95^{\mathrm{a}}$ & \\
\hline
\end{tabular}

Figures with different letters are significant $(\mathrm{p}<0.05)$

Relatively, however, Shinoda et al. (2004) claimed that sugars had little effect on browning at early stage of storage of model OJ solution, but browning was more stimulated in later stage of storage. While there was no significant alteration in $\mathrm{pH}$ mean values of both OJ samples stored at $4{ }^{\circ} \mathrm{C}$ and $20{ }^{\circ} \mathrm{C}$, they showed a significant $\mathrm{pH}$ difference $(\mathrm{p}<0.05)$ as compared with the fresh squeezed OJ. This can be attributed by the degradation products of AA and RS through Maillard reaction. 


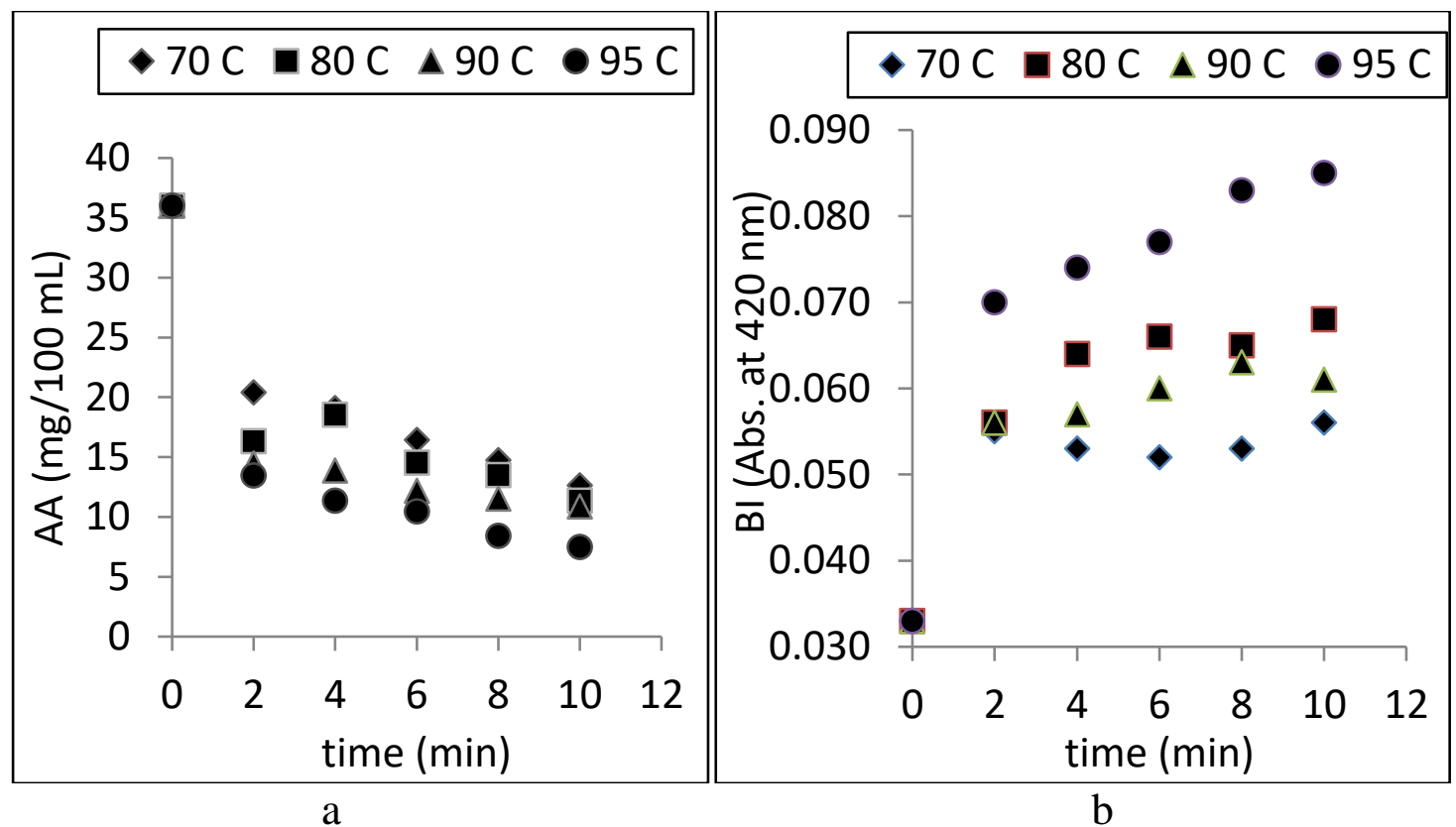

Figure (1) Heat treatment profiles at time zero affecting ascorbic acid content (a) and browning index (b) of orange juice

\section{Microwave treatment of orange juice samples}

Table 5 describes the effect of MW treatment and storage conditions on the parameters studied of OJ samples. It can be noticed that the AA of juice samples at T0 (at range between 20.82-36 mg/100 mL) has been subjected to less effect by MW treatment rather than to heat treatment, however the higher time consuming of MW treatment caused higher reduction in AA content (Table 5). Later observation can be related to the temperature increased $\left(37-98{ }^{\circ} \mathrm{C}\right)$ as microwaving time increased (30-150 sec.). This was also speculated by Vikram et al. (2005) who referred to the ascorbic degradation mainly to the internal heat generation under MW heating. Nevertheless, the mean value of AA at T0 $(26.98 \mathrm{mg} / 100 \mathrm{~mL})$ showed a significant reduction $(\mathrm{p}<0.05)$ as a comparison with initial AA content $(36 \mathrm{mg} / 100$ $\mathrm{mL})$. It was also stated that the MW treatment has monitored a decrease of AA in OJ (Cinquanta et al., 2010). However, Igual et al. (2010) reported that AA retention in grapefruit juice was superior in the MW treatment than in the conventional treatment. In this sense, the use of MW energy seems to cause lesser changes in the fruit quality attributes (Nikdel et al., 1993).

Overall mean values showed significant decrease $(\mathrm{p}<0.05)$ in AA after 2 month storage at both 4 and $20^{\circ} \mathrm{C}$, however, AA retention was higher at $4{ }^{\circ} \mathrm{C}$ than at $20{ }^{\circ} \mathrm{C}$ storage temperatures (Table 5). According to the published data, the content of $\mathrm{AA}$ in different juices decreases during storage, depending on temperature, oxygen, and light access (Klimczak et al., 2007). Nevertheless, microwaving for $30 \mathrm{sec}$. gave the lowest AA $(\mathrm{p}<0.05)$ in samples stored for 2 months at $4{ }^{\circ} \mathrm{C}$ and $20{ }^{\circ} \mathrm{C}$ in comparison with other stored samples processed at different MW conditions (Fig. 3a). This could be attributed to insufficient effect of such specific MW treatment $(30 \mathrm{sec}$.) which might not be enough to eliminate the deteriorating effects such as microbial growth (van Boekel, 2002) and/or biological 
Mesopotamia J. of Agric.

Vol. (46) No. (3) 2018

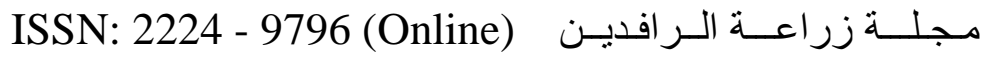
ISSN: 1815 - 316 X (Print) 2018 المجلا (46) العدد (3)

Table (3) Time and temperature effects on fresh orange juice properties after 2 months of storage at $4{ }^{\circ} \mathrm{C}$

\begin{tabular}{|c|c|c|c|c|c|c|}
\hline \multirow[t]{2}{*}{ Determination } & \multirow[t]{2}{*}{ Time (min) } & \multicolumn{4}{|c|}{ Temperature $\left({ }^{\circ} \mathrm{C}\right)$} & \multirow[t]{2}{*}{ Mean } \\
\hline & & 70 & 80 & 90 & 95 & \\
\hline \multirow{6}{*}{$\begin{array}{l}\text { Ascorbic acid } \\
(\mathrm{mg} / 100 \mathrm{~mL})\end{array}$} & 2 & 19.91 & 15.88 & 13.57 & 12.43 & $15.45^{\mathrm{a}}$ \\
\hline & 4 & 18.59 & 16.93 & 13.21 & 11.87 & $15.15^{\mathrm{a}}$ \\
\hline & 6 & 15.44 & 13.41 & 11.40 & 9.90 & $12.54^{b}$ \\
\hline & 8 & 14.73 & 12.71 & 10.68 & 8.18 & $11.58^{\mathrm{c}}$ \\
\hline & 10 & 13.41 & 10.59 & 8.52 & 7.58 & $10.08^{\mathrm{d}}$ \\
\hline & Mean & $16.46^{\mathrm{a}}$ & $13.90^{\mathrm{b}}$ & $11.48^{\mathrm{c}}$ & $9.99^{d}$ & \\
\hline \multirow{6}{*}{$\begin{array}{l}\text { Browning index } \\
\text { (Abs. at } 420 \mathrm{~nm} \text { ) }\end{array}$} & 2 & 0.112 & 0.116 & 0.124 & 0.128 & $0.120^{\mathrm{c}}$ \\
\hline & 4 & 0.115 & 0.118 & 0.125 & 0.125 & $0.121^{\mathrm{c}}$ \\
\hline & 6 & 0.112 & 0.114 & 0.126 & 0.133 & $0.121^{\mathrm{c}}$ \\
\hline & 8 & 0.114 & 0.126 & 0.153 & 0.141 & $0.133^{b}$ \\
\hline & 10 & 0.121 & 0.127 & 0.153 & 0.190 & $0.148^{\mathrm{a}}$ \\
\hline & Mean & $0.115^{\mathrm{a}}$ & $0.120^{\mathrm{b}}$ & $0.136^{\mathrm{c}}$ & $0.143^{d}$ & \\
\hline \multirow{6}{*}{$\begin{array}{l}\text { Reducing sugar } \\
(\mathrm{gm} / 100 \mathrm{~mL})\end{array}$} & 2 & 4.71 & 4.84 & 4.90 & 4.90 & $4.84^{\mathrm{a}}$ \\
\hline & 4 & 4.65 & 4.79 & 4.47 & 4.82 & $4.68^{\mathrm{ab}}$ \\
\hline & 6 & 4.63 & 4.62 & 4.46 & 4.55 & $4.57^{b}$ \\
\hline & 8 & 4.41 & 4.55 & 4.45 & 4.46 & $4.47^{b}$ \\
\hline & 10 & 4.40 & 3.42 & 4.26 & 4.39 & $4.13^{c}$ \\
\hline & Mean & $4.56^{\mathrm{a}}$ & $4.44^{\mathrm{a}}$ & $4.51^{\mathrm{a}}$ & $4.63^{\mathrm{a}}$ & \\
\hline \multirow{6}{*}{$\begin{array}{l}\text { Total soluble solids } \\
\qquad(\%)\end{array}$} & 2 & 10.2 & 10.5 & 10.4 & 10.4 & $10.4^{\mathrm{c}}$ \\
\hline & 4 & 10.4 & 10.5 & 10.5 & 10.5 & $10.5^{b c}$ \\
\hline & 6 & 10.5 & 10.6 & 10.6 & 10.6 & $10.6^{b}$ \\
\hline & 8 & 10.6 & 10.6 & 10.8 & 10.9 & $10.7^{\mathrm{a}}$ \\
\hline & 10 & 10.6 & 10.8 & 10.8 & 10.9 & $10.8^{\mathrm{a}}$ \\
\hline & Mean & $10.5^{b}$ & $10.6^{\mathrm{a}}$ & $10.6^{\mathrm{a}}$ & $10.7^{\mathrm{a}}$ & \\
\hline \multirow{6}{*}{$\mathrm{pH}$} & 2 & 2.99 & 2.88 & 3.05 & 3.08 & $3.00^{\mathrm{a}}$ \\
\hline & 4 & 2.99 & 2.95 & 3.02 & 3.02 & $3.00^{\mathrm{a}}$ \\
\hline & 6 & 2.99 & 2.99 & 3.02 & 3.01 & $3.00^{\mathrm{a}}$ \\
\hline & 8 & 2.99 & 3.00 & 3.00 & 3.01 & $3.01^{\mathrm{a}}$ \\
\hline & 10 & 2.99 & 3.00 & 3.00 & 3.02 & $3.00^{\mathrm{a}}$ \\
\hline & Mean & $2.99^{b}$ & $2.97^{b}$ & $3.03^{\mathrm{a}}$ & $3.03^{\mathrm{a}}$ & \\
\hline
\end{tabular}

Figures with different letters are significant $(\mathrm{p}<0.05)$ 
Mesopotamia J. of Agric.

Vol. (46) No. (3) 2018

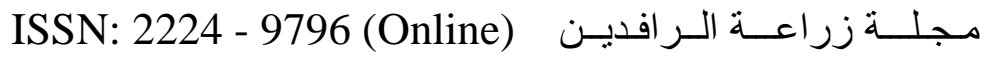
ISSN: 1815 - 316 X (Print) 2018 الهجلد (46) العدد (3)

Table (4) Time and temperature effects on fresh orange juice properties after 2 months of storage at $20^{\circ} \mathrm{C}$

\begin{tabular}{|c|c|c|c|c|c|c|}
\hline \multirow[t]{2}{*}{ Determination } & \multirow[t]{2}{*}{ Time(min) } & \multicolumn{4}{|c|}{ Temperature $\left({ }^{\circ} \mathrm{C}\right)$} & \multirow[t]{2}{*}{ Mean } \\
\hline & & 70 & 80 & 90 & 95 & \\
\hline \multirow{6}{*}{$\begin{array}{l}\text { Ascorbic acid } \\
(\mathrm{mg} / 100 \mathrm{~mL})\end{array}$} & 2 & 14.47 & 5.77 & 5.78 & 3.33 & $7.34^{\mathrm{a}}$ \\
\hline & 4 & 13.56 & 5.62 & 5.77 & 3.82 & $7.14^{\mathrm{a}}$ \\
\hline & 6 & 12.13 & 5.24 & 5.21 & 3.62 & $6.49^{a}$ \\
\hline & 8 & 12.04 & 5.13 & 5.11 & 3.65 & $6.48^{\mathrm{a}}$ \\
\hline & 10 & 9.33 & 4.83 & 4.42 & 3.37 & $5.49^{\mathrm{b}}$ \\
\hline & Mean & $12.31^{\mathrm{a}}$ & $5.32^{b}$ & $5.26^{\mathrm{b}}$ & $3.47^{c}$ & \\
\hline \multirow{6}{*}{$\begin{array}{l}\text { Browning index } \\
\text { (Abs. at } 420 \mathrm{~nm} \text { ) }\end{array}$} & 2 & 0.163 & 0.253 & 0.249 & 0.296 & $0.240^{\mathrm{c}}$ \\
\hline & 4 & 0.169 & 0.256 & 0.253 & 0.292 & $0.243^{\mathrm{c}}$ \\
\hline & 6 & 0.180 & 0.263 & 0.261 & 0.297 & $0.250^{\mathrm{b}}$ \\
\hline & 8 & 0.181 & 0.263 & 0.267 & 0.291 & $0.251^{\mathrm{b}}$ \\
\hline & 10 & 0.192 & 0.269 & 0.276 & 0.301 & $0.260^{\mathrm{a}}$ \\
\hline & Mean & $0.177^{\mathrm{c}}$ & $0.261^{\mathrm{b}}$ & $0.261^{\mathrm{b}}$ & $0.295^{\mathrm{a}}$ & \\
\hline \multirow{6}{*}{$\begin{array}{l}\text { Reducing sugar } \\
(\mathrm{gm} / 100 \mathrm{~mL})\end{array}$} & 2 & 4.91 & 4.83 & 4.76 & 4.65 & $4.79^{\mathrm{a}}$ \\
\hline & 4 & 4.49 & 4.81 & 4.76 & 4.62 & $4.67^{b}$ \\
\hline & 6 & 4.36 & 4.52 & 4.74 & 4.43 & $4.51^{\mathrm{c}}$ \\
\hline & 8 & 4.44 & 4.38 & 4.65 & 4.31 & $4.46^{\mathrm{cd}}$ \\
\hline & 10 & 4.38 & 4.29 & 4.60 & 4.27 & $4.38^{\mathrm{d}}$ \\
\hline & Mean & $4.53^{\mathrm{bc}}$ & $4.57^{\mathrm{b}}$ & $4.70^{\mathrm{a}}$ & $4.46^{c}$ & \\
\hline \multirow{6}{*}{$\begin{array}{l}\text { Total soluble } \\
\text { solids (\%) }\end{array}$} & 2 & 10.6 & 10.6 & 10.5 & 10.8 & $10.6^{\mathrm{d}}$ \\
\hline & 4 & 10.7 & 10.6 & 10.5 & 10.8 & $10.7^{\mathrm{cd}}$ \\
\hline & 6 & 10.9 & 10.7 & 10.5 & 10.9 & $10.8^{\mathrm{c}}$ \\
\hline & 8 & 11.1 & 10.9 & 10.6 & 10.9 & $10.9^{\mathrm{b}}$ \\
\hline & 10 & 11.3 & 11.1 & 10.8 & 11.2 & $11.1^{\mathrm{a}}$ \\
\hline & Mean & $10.9^{\mathrm{a}}$ & $10.8^{\mathrm{a}}$ & $10.6^{\mathrm{b}}$ & $10.9^{c}$ & \\
\hline \multirow{6}{*}{$\mathrm{pH}$} & 2 & 2.99 & 3.02 & 3.03 & 3.02 & $3.02^{b}$ \\
\hline & 4 & 2.99 & 3.02 & 3.03 & 3.00 & $3.01^{\mathrm{b}}$ \\
\hline & 6 & 3.00 & 3.02 & 3.01 & 3.01 & $3.01^{\mathrm{b}}$ \\
\hline & 8 & 3.00 & 3.03 & 3.00 & 3.03 & $3.02^{\mathrm{b}}$ \\
\hline & 10 & 3.01 & 3.06 & 3.07 & 3.00 & $3.04^{\mathrm{a}}$ \\
\hline & Mean & $3.00^{b}$ & $3.03^{a}$ & $3.03^{a}$ & $3.01^{b}$ & \\
\hline
\end{tabular}

Figures with different letters are significant $(\mathrm{p}<0.05)$ 
Mesopotamia J. of Agric.

Vol. (46) No. (3) 2018
ISSN: 2224 - 9796 (Online) مجلــة زر اعــة الـر افديـن ISSN: 1815 - 316 X (Print) 2018 المجلد (46) العدد (3)

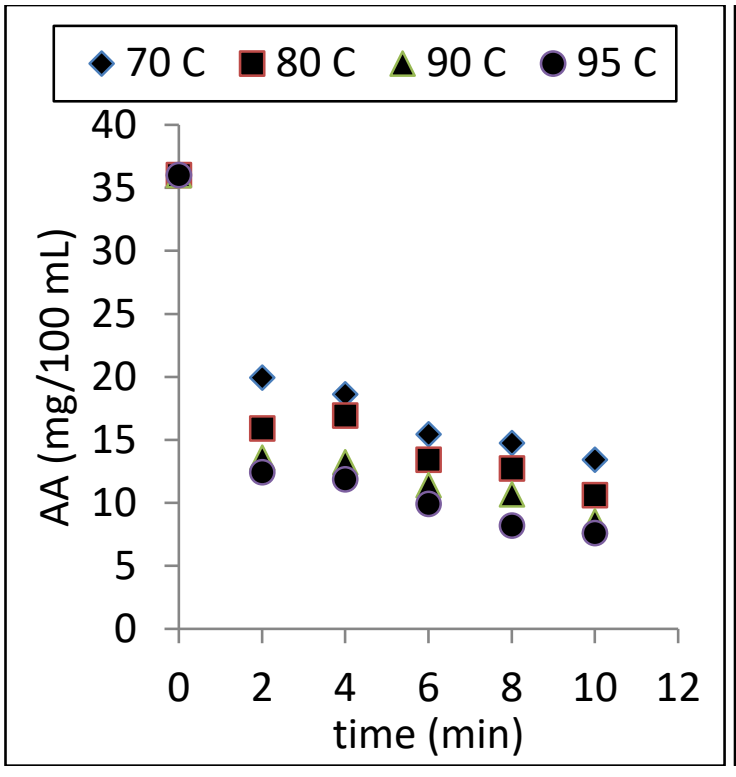

a

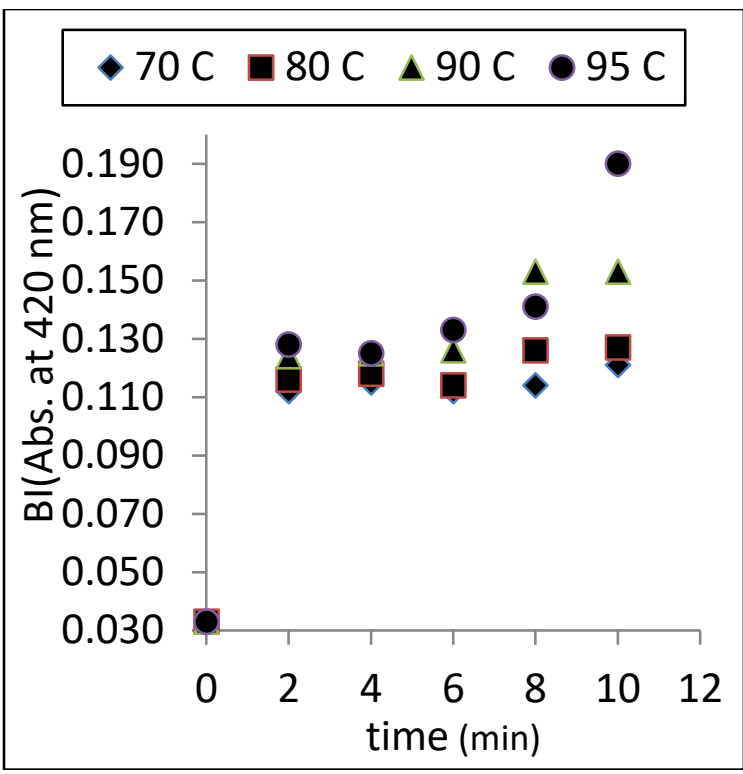

b

Figure (2) Heat treatment profiles for 2 months storage at $4{ }^{\circ} \mathrm{C}$ affecting ascorbic acid content (a) and browning index (b) of orange juice

Table (5) Effect of microwave at $2450 \mathrm{MHz}$ for different durations on fresh orange juice properties

\begin{tabular}{|c|c|c|c|c|c|c|c|}
\hline \multirow{2}{*}{ Determination } & \multirow[b]{2}{*}{ Storage time } & \multicolumn{5}{|c|}{ Microwaving time (sec.) } & \multirow[b]{2}{*}{ Mean } \\
\hline & & 30 & 60 & 90 & 120 & 150 & \\
\hline \multirow{3}{*}{$\begin{array}{l}\text { Ascorbic acid } \\
(\mathrm{mg} / 100 \mathrm{~mL})\end{array}$} & Time zero & 36.00 & 26.53 & 26.29 & 25.24 & 20.82 & $26.98^{\mathrm{a}}$ \\
\hline & 2 months at $4{ }^{\circ} \mathrm{C}$ & 14.27 & 20.11 & 25.16 & 24.33 & 19.52 & $20.68^{b}$ \\
\hline & 2 months at $20^{\circ} \mathrm{C}$ & 10.20 & 15.33 & 16.71 & 16.30 & 21.67 & $16.04^{\mathrm{c}}$ \\
\hline \multirow{3}{*}{$\begin{array}{l}\text { Browning index } \\
\text { (Abs. at 420nm) }\end{array}$} & Time zero & 0.052 & 0.049 & 0.051 & 0.058 & 0.058 & $0.053^{\mathrm{c}}$ \\
\hline & 2 months at $4{ }^{\circ} \mathrm{C}$ & 0.161 & 0.148 & 0.139 & 0.118 & 0.101 & $0.134^{b}$ \\
\hline & 2 months at $20^{\circ} \mathrm{C}$ & 0.324 & 0.276 & 0.238 & 0.226 & 0.198 & $0.252^{\mathrm{a}}$ \\
\hline \multirow{3}{*}{$\begin{array}{l}\text { Reducing Sugar } \\
(\mathrm{gm} / 100 \mathrm{~mL})\end{array}$} & Time zero & 4.80 & 5.00 & 4.90 & 4.90 & 5.00 & $4.92^{\mathrm{a}}$ \\
\hline & 2 months at $4{ }^{\circ} \mathrm{C}$ & 1.98 & 4.11 & 4.56 & 4.76 & 4.92 & $4.07^{b}$ \\
\hline & 2 months at $20^{\circ} \mathrm{C}$ & 0.79 & 2.79 & 4.38 & 4.68 & 4.93 & $3.51^{b}$ \\
\hline \multirow{3}{*}{$\begin{array}{l}\text { Total soluble } \\
\text { solids }(\%)\end{array}$} & Time zero & 10.5 & 10.4 & 10.4 & 10.8 & 10.8 & $10.6^{\mathrm{a}}$ \\
\hline & 2 months at $4{ }^{\circ} \mathrm{C}$ & 9.4 & 9.5 & 10.0 & 10.1 & 10.1 & $9.80^{b}$ \\
\hline & 2 months at $20^{\circ} \mathrm{C}$ & 7.1 & 9.1 & 9.8 & 10.2 & 10.4 & $9.32^{c}$ \\
\hline \multirow{3}{*}{$\mathrm{pH}$} & Time zero & 2.92 & 2.93 & 2.93 & 2.94 & 2.94 & $2.93^{b}$ \\
\hline & 2 months at $4{ }^{\circ} \mathrm{C}$ & 2.91 & 2.91 & 2.93 & 2.97 & 2.99 & $2.94^{b}$ \\
\hline & 2 months at $20^{\circ} \mathrm{C}$ & 2.99 & 3.01 & 3.01 & 3.02 & 3.03 & $3.01^{\mathrm{a}}$ \\
\hline
\end{tabular}

Figures with different letters are significant $(\mathrm{p}<0.05)$

interaction (i.e. enzymatic activity)(Ceni et al., 2009) stimulating AA degradation in juice samples. However, Ceni et al. (2009) showed that a better result of inactivation of polyphenols oxidase and peroxidase was only obtained where MW exposure reached at higher than $60 \mathrm{sec}$. Nevertheless, the mean value of AA achieved at T0 $(26.98 \mathrm{mg} / 100 \mathrm{~mL})$ (Table 5)were significantly higher $(\mathrm{p}<0.05)$ than those recorded for heat-treated samples for time and temperature used in the range of 10.57-16.16 
and 10.22-16.66 mg/100 mL respectively (Table 1). Browning index values, considered as a reflection of browning colour, were increased $(\mathrm{p}<0.05)$ at $\mathrm{T} 0$, although the MW exposure for 120 and $150 \mathrm{sec}$. recorded the highest BI values (Table 5). On the other word, the samples exposed to MW energy for a longer time presented minor increase of $\mathrm{BI}$ difference. This can be attributed to higher temperatures (at the range of $37-98{ }^{\circ} \mathrm{C}$ ) occurred with higher MW exposure times (at the range of 30-150 sec.) used. Heating can induce undesirable changes in colour, flavour, and nutritional value of OJ (Giner et al., 2003). However, MW effect at $\mathrm{T} 0$ showed less BI mean value (0.053) than BI mean values stimulated by various times and temperatures used in heat treatments, in the range of 0.059-0.067 and 0.054-0.078 respectively (Table 1). Regarding the BI as a reflection of browning colour intensity, the higher value of BI was coincided with using higher MW exposure time (120-150 sec.) of which this can somehow related to the higher degradation of $\mathrm{AA}$ at this exposure time range. As indicated previously that the ascorbic acid degradation products is one of the vital browning colour production in fruit juiced (Ibarz et al., 1990).

In spite of overall increasing in BI of juice samples $(p<0.05)$ stored for 2 months, microwave processed samples stored at $4{ }^{\circ} \mathrm{C}$ showed less $\mathrm{BI}$ mean value (0.134) than those stored at $20^{\circ} \mathrm{C}(0.252)$ (Table 5). However, juice samples MWprocessed at 120 and $150 \mathrm{sec}$. gave the least BI values when they were stored at $4{ }^{\circ} \mathrm{C}$ for 2 months (Fig. 3b). Later observation suggests that MW exposure for 120$150 \mathrm{sec}$. and storage at $4{ }^{\circ} \mathrm{C}$ dragging the deterioration reaction to produce browning colour. Similarly, Igual et al. (2010) reported a similar observation with MW treatment of grapefruit juice.

Microwave treatment seems to have little effect on RS content, TSS \%, and $\mathrm{pH}$ of juice samples at $\mathrm{T} 0$ in comparison with samples stored at both storage temperatures 4 and $20{ }^{\circ} \mathrm{C}$ for 60 days (Table 5), although, from the statistical analysis, their mean values showed a significant reduction $(\mathrm{p}<0.05)$ from their initial values. These quality parameters are important as they are closely related with the stability of the bioactive compounds in fruit products (Snchez-Moreno et al., 2006). Similar results were obtained at day 1 when MW treatment implemented on grapefruit juice (Igual et al., 2010). Nevertheless, these parameters expressed different depression as they stored for 60 days at $4{ }^{\circ} \mathrm{C}$ and $20{ }^{\circ} \mathrm{C}$ especially with AA and BI values (Fig. 3a,b). Although for storing condition at $4{ }^{\circ} \mathrm{C}$ showed less reduction in $\mathrm{RS}$, TSS $\%$, and $\mathrm{pH}$, they were significantly different $(\mathrm{p}<0.05)$ from either the initial fresh juice samples or samples MW-processed at T0. In contrary, these parameters (RS values in particular) of juice processed samples at MW exposure of $30 \mathrm{sec}$. and stored at different conditions illustrated the lowest values. As stated above that MW exposure for $30 \mathrm{sec}$. was probably insufficient electromagnetic level for lethal contribution of microbial inactivation and eliminating the biological interaction. 
Mesopotamia J. of Agric.

Vol. (46) No. (3) 2018

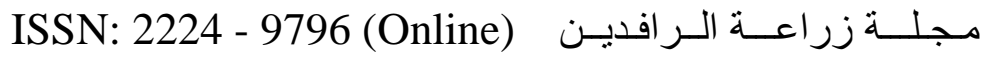
ISSN: 1815 - 316 X (Print) 2018 المجلد (46) العدد (3)

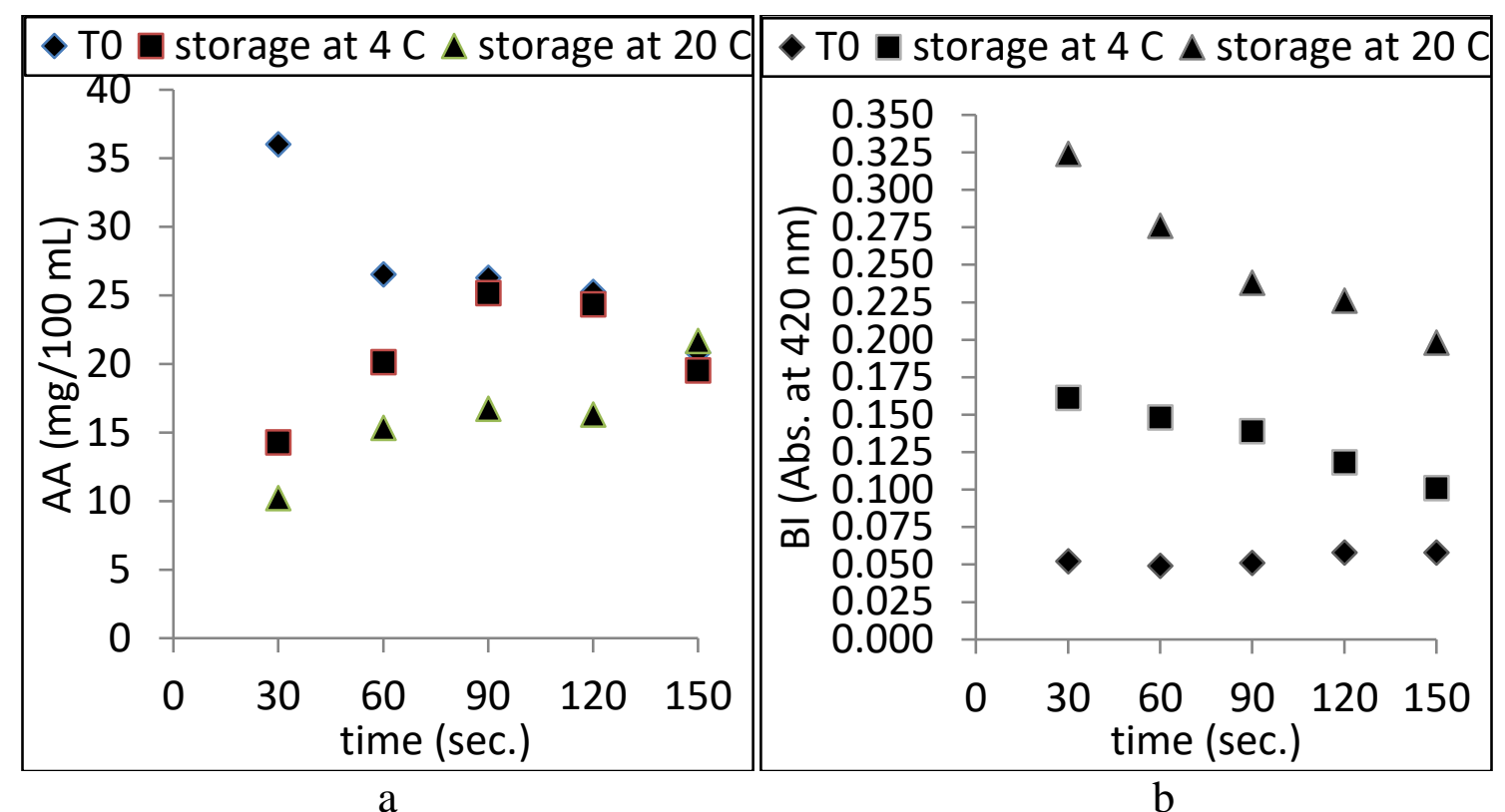

Figure (3) Microwave treatment effect on some orange juice properties at time zero and after 2 month storage at $4{ }^{\circ} \mathrm{C}$ and $20{ }^{\circ} \mathrm{C}$ as ascorbic acid (a) and browning index (b)

Generally speaking, using MW process has shown promising results for improving product quality and, particularly for shortening the treatment times. Contrary to conventional treatment, MW treatment showed better values particularly in AA which considered to be the nutritional attraction factor for consumer acceptability. Moreover, the storage of refrigerated heat and MW-treated OJ samples at $4{ }^{\circ} \mathrm{C}$ were more stable in preserving the parameters studied rather than those stored at $20{ }^{\circ} \mathrm{C}$. However, MW exposure time should exceed $60 \mathrm{sec}$. because of using less exposure time was not enough to preserve the OJ quality. Since this study with OJ was conducted at laboratory scale with a domestic MW oven, studies on large sample sizes may be required for use of MW energy as an alternative to the stage at commercial scale.

\section{تأثير المعاملات الحرارية والموجات الدقيقة على نوعية عصير البرتقال أثناء الذزن$$
\text { ثامر عبد القادر خليل }
$$$$
\text { قسم علوم الأغذية، كلية الزراعة والغابات، جامعة الموصل/ العراق. }
$$$$
\text { Emil:thamerkhalil@yahoo.com }
$$

\footnotetext{
الخلاصة

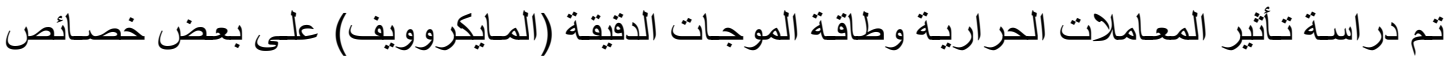

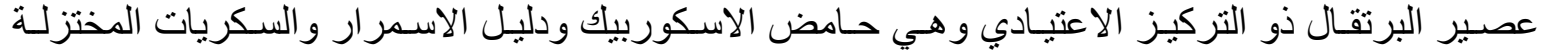

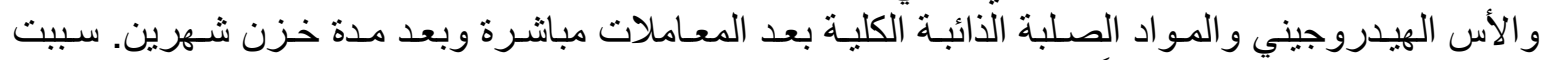

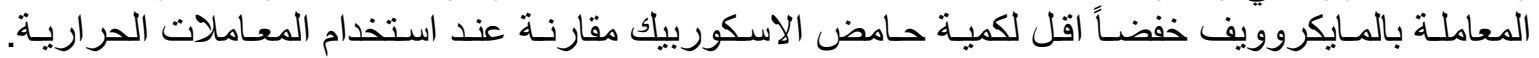

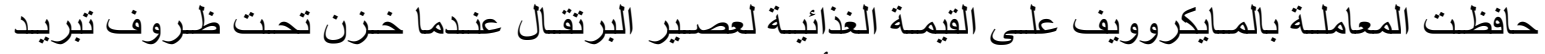

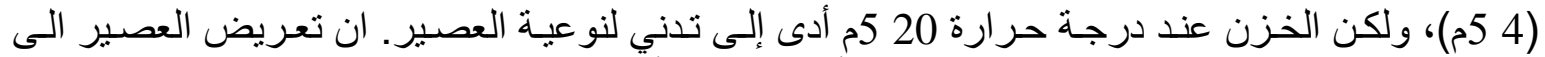

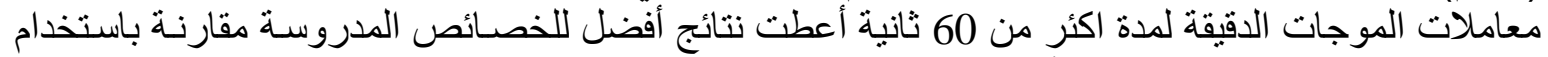

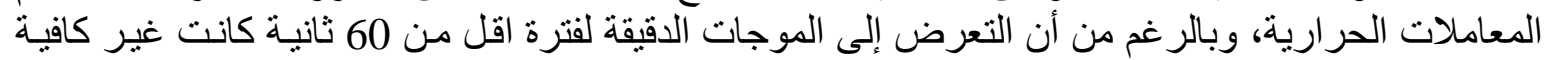


للحفاظ على نوعية عصبر البرتقال. من خلال هذه النتائج، يمكن استخدام طاقة الموجات الدقيقة لتكون بديل اللمعاملات الحرارية التقليدية للحفاظ على نوعية عصلير البرتية لنقال.

\section{REFERENCES}

Anonymous (1997). Statistical Analysis System (SAS). Institute Inc. Proprietary software release 6.12 TS020. Cary, North Carolina State University, USA.

Beveridge, T.; K. Y. Franz and J. E. Harrison (1986). Clarified natural apple juice: Production and storage stability of juice and concentrate. Journal of Food Science 51: 411-414.

Braddock, R. J. (1999). Handbook of Citrus By-products and Treatment Technology. Wiley, New York.

Burdurlu, H. S. and F. F. Karadeniz (2003). Effect of storage on nonenzymatic browning of apple juice concentrates. Food Chemistry 80: 91-97.

Canumir, J. A.; J. E. Celis; J. Brujin and L. Vidal (2002). Pasteurization of apple juice by using microwaves. Lebensmittel-Wissenschaft und Technologie (35) 5: 389-392.

Ceni G. C.; E. M. Baldissera; M. D. S. Primo; O. A. C. Antunes; C. Dariva; J. V. D. Oliveira and D. D. Oliveira (2009). Influence of application of microwave energy on quality parameters of mate tea leaves (Ilex paraguariensis St. Hil.). Food Technology and Biotechnology 47 (2): 221-226.

Cinquanta, L.; D. Albanese; G. Cuccurullo and M. Di Matteo (2010). Effect on orange juice of batch pasteurization in an improved pilot-scale microwave oven. Journal of Food Science 75 (1): 46-50.

Cornwell, C. J. and R. E. Wrolstad (1981). Causes of browning in pear juice concentrate during storage. Journal of Food Science 46: 515-518.

Damasceno, L. F.; F. A. N. Fernandes; M. M. A. Magalhães and E. S. Brito (2008). Evaluation and optimization of nonenzymatic browning of "CAJUINA" during thermal treatment. Brazilian Journal of Chemical Engineering 25 (2): 313-320.

Dio Alvarado, J. and N. Viteri (1991). Effect of temperature on the aerobic degradation of Vitamin C in citric fruit juices. Archives Latino Nutrition 39: 601-612.

Elez-Martınez, P.; R. C. Soliva-Fortuny and O. Martın-Belloso (2006). Comparative study on shelf life of orange juice processed by high intensity pulsed electric fields or heat treatment. European Food Research and Technology 222: 321-329.

Giner, J.; V. Gimeno; M. Palom'es ; G. V Barbosa-Canovas and O. Martın (2003). Lessening polygalacturonase activity in a commercial enzyme preparation by exposure to pulsed electric fields. European Food Research Technology 217:43-48.

Handwerk, R. L. and R. L. Coleman (1988). Approaches to the citrus browning problem, A review. Journal of Agricalture and Food Chemistry 36: 231-236. 
Ibarz, I.; C. Gonzles; S. Esplugas and R. Meguelsanz (1990). Nonenzymatic browning kinetics of clarified peach juice at different temperatures. Confructa 34: 152-59.

Igual, M.; E. Garcia-Martinez; M. M. Camacho and N. Martinez-Navarrete (2010). Effect of thermal treatment and storage on the stability of organic acids and the functional value of grapefruit juice. Food Chemistry 118: 291-299.

Khalil, T. A. and M. I. Al-Zubaidy (2010). Kinetics of nonenzymatic browning reaction in citrus juice concentrates during storage. $4^{\text {th }}$ International Conference, "Innovative in Food Science and Nutrition: Future Challenges", NRC, Cairo, 27-29 Sep. 2010.

Kim, H. B. and C. C. Tadini (1999). Effect of different pasteurization conditions on enzyme inactivation of orange juice in pilot-scale experiments. Journal of Food Process Engineering 22: 395-403.

Klimczak, I.; M. Malecka; M. Szlachta and A. Gliszcynska (2007). Effect of storage on the content of polyphenols, vitamin $\mathrm{C}$ and the antioxidant activity of orange juices. Journal of Food Compositional Analysis 20: 313-322.

Kozempel, M. F.; R. D. Cook; O. J. Scullen and B. A. Annous (2000). Development of a process for detecting nonthermal effects of microwave energy on microorganisms at low temperature. Journal of Food Process and Preservation 24: 287-301.

Lima, M.; B. F. Heskitt; L. L. Burianek; S. E. Nokes and S. K. Sastry (1999). Ascorbic acid degradation kinetics during conventional and ohmic heating. Journal of Food Process and Preservation 23: 421-434.

Metaxas, A. C. (1996). Foundations of Electroheat: a Unified Approach, John Wiley and Sons.

Metaxas, A. C. and R. J. Meredith (1993). Industrial Microwave Heating. Peter Peregrinus, United Kingdom.

Miller, G. L. (1972). Use of dinitrosalicylic acid reagent for determination of reducing sugars. Analytical Chemistry 31: 426-428.

Nikdel, S.; C. Chen; M. Parish; D. MacKellar and L. Friedrich (1993). Pasteurization of citrus juice with microwaves energy in a continuous-flow unit. Journal of Agriculture Food Chemistry 41: 2116-2119.

Ranganna, S. (1977). Manual of Analysis of Fruit and Vegetable Products. Taha McGraw-Hill, New Delhi.

Revanasiddappa, H. D. and M. A. Veena (2008). Sensitive spectrophotometric methods for the determination of ascorbic acid. Electrical Journal of Chemistry (5) 1: 10-15.

Shinoda, Y.; M. Murata; S. Homma and H. Komura (2004). Browning and decomposed products of model orange juice. Bioscience, Biotechnology and Biochemistry 68 (3): 529-536.

Snchez-Moreno, C.; L. Plaza; B. De Ancos and M. P. Cano (2006). Nutritional characterization of commercial traditional pasteurized tomato juices: Carotenoids, vitamin $\mathrm{C}$ and radical-scavenging capacity. Food Chemistry 98: 749-756.

Snir, R.; P. E. Koehler; K. A. Sims and L. Wicker (1996). Total and thermostable pectinesterases in citrus juices. Journal of Food Science 61 (2): 379-382. 
Tatum, J. H.; P. E. Shaw and R. E. Berry (1969). Degradation products from ascorbic acid. Journal of Agriculture and Food Chemistry 17: 38-40.

Tatum, J. H.; S. Nagy and R. E. Berry (1975). Degradation products formed in canned single-strength orange juice during storage. Journal of Food Science 40: 707-709.

Van Boekel, M. A. J. S. (2002). On the use of the Weibull model to describe thermal inactivation of microbial vegetative cells. International Journal of Food Microbiology 74: 139-59.

Vikram V. B.; M. N. Ramesh and S. G. Prapulla (2005). Thermal degradation kinetics of nutrients in orange juice heated by electromagnetic and conventional methods. Journal of Food Engineering 69 (1): 31-40. 